\title{
Influence of Particle Size of Deproteinized Bovine Bone Mineral on New Bone Formation and Implant Stability after Simultaneous Sinus Floor Elevation: A Histomorphometric Study in Minipigs
}

\author{
Simon S. Jensen, DDS; ${ }^{*}$ Merete Aaboe, DDS, PhD; ${ }^{\dagger}$ Simone F. M. Janner, DDS, Dr med. dent; \\ Nikola Saulacic, DDS, PhD; ${ }^{\S}$ Michael M. Bornstein, DDS, PD; ${ }^{\Im}$ Dieter D. Bosshardt, DMD, PhD;** \\ Daniel Buser, DDS, Dr med dent. ${ }^{\dagger \dagger}$
}

\begin{abstract}
Background: Deproteinized bovine bone mineral (DBBM) is one of the best-documented bone substitute materials for sinus floor elevation (SFE).

Purpose: DBBM is available in two particle sizes. Large particles are believed to facilitate improved neoangiogenesis compared with small ones. However, their impact on the rate of new bone formation, osteoconduction, and DBBM degradation has never been reported. In addition, the implant stability quotient (ISQ) has never been correlated to bone-to-implant contact (BIC) after SFE with simultaneous implant placement.
\end{abstract}

Materials and Methods: Bilateral SFE with simultaneous implant placement was performed in 10 Göttingen minipigs. The two sides were randomized to receive large or small particle size DBBM. Two groups of 5 minipigs healed for 6 and 12 weeks, respectively. ISQ was recorded immediately after implant placement and at sacrifice. Qualitative histological differences were described and bone formation, DBBM degradation, BIC and bone-to-DBBM contact (osteoconduction) were quantified histomorphometrically.

Results: DBBM particle size had no qualitative or quantitative impact on the amount of newly formed bone, DBBM degradation, or BIC for either of the healing periods $(p>0.05)$. Small-size DBBM showed higher osteoconduction after 6 weeks than large-size DBBM $(p<0.001)$. After 12 weeks this difference was compensated. There was no significant correlation between BIC and ISQ.

Conclusion: Small and large particle sizes were equally predictable when DBBM was used for SFE with simultaneous implant placement.

KEY WORDS: animal study, bone substitutes, histological analysis, implant stability, sinus floor elevation

${ }^{*}$ Senior lecturer, Department of Oral Surgery and Stomatology, School of Dental Medicine, University of Bern, Bern, Switzerland and consultant oral and maxillofacial surgeon, Department of Oral and Maxillofacial Surgery, Copenhagen University Hospital (Rigshospitalet), Copenhagen, Denmark; ${ }^{\dagger}$ associate professor, Department of Oral and Maxillofacial Surgery and Oral Pathology, School of Dentistry, Health, Aarhus University, Aarhus, Denmark; ${ }^{\ddagger}$ Department of Oral Surgery and Stomatology, School of Dental Medicine, University of Bern, Bern, Switzerland; 'research associate, Department of Oral Surgery and Stomatology, School of Dental Medicine, University of Bern, Bern, Switzerland, and Department of Cranio-Maxillofacial Surgery, Bern University Hospital, Bern, Switzerland; 'assistant professor, Department of Oral Surgery and Stomatology, School of Dental Medicine, University of Bern, Bern, Switzerland; ${ }^{* *}$ professor, Department of Oral Surgery and Stomatology, School of Dental Medicine, University of Bern, Bern, Switzerland, and Robert K. Schenk Laboratory of Oral Histology, School of Dental Medicine,

\section{INTRODUCTION}

Ongoing pneumatization of the maxillary sinus in the edentulous or partially edentulous posterior maxilla

University of Bern, Bern, Switzerland; ${ }^{\dagger \dagger}$ professor, Department of Oral Surgery and Stomatology, School of Dental Medicine, University of Bern, Bern, Switzerland

Reprint requests: Dr. Simon Storgård Jensen, Department of Oral and Maxillofacial Surgery, Copenhagen University Hospital (Rigshospitalet), Blegdamsvej 9, DK-2100 Copenhagen $\varnothing$, Denmark; e-mail: simon.storgaard@jensen.mail.dk

(C) 2013 Wiley Periodicals, Inc.

DOI $10.1111 /$ cid.12101 
often reduces the residual subantral bone height considerably. As a result, placement of implants of sufficient length may be compromised. Placement of short implants has been suggested as an alternative to bone regeneration procedures in the atrophic posterior maxilla. ${ }^{1}$ However, implants of $10 \mathrm{~mm}$ or more are recommended to increase the probability of long-term implant survival. ${ }^{2}$ Therefore, maxillary sinus floor elevation (SFE) is often indicated before or simultaneously with implant insertion in the posterior maxilla. SFE may be performed using a lateral window or transcrestal approach. ${ }^{3}$ With both techniques, the sinus membrane (also referred to as the schneiderian membrane) is carefully elevated in a cranial direction. The created volume is most often filled with a bone grafting material. The most frequently encountered grafting protocols include autogenous bone from intra- or extraoral donor sites, a bone substitute material or combinations thereof (i.e., composite grafts). ${ }^{3}$

Autogenous bone grafts used alone are associated with various degrees of resorption. ${ }^{4-6}$ In addition, the harvesting procedure may be accompanied by significant morbidity and complications at the donor site especially when autografts are harvested extraorally or intraorally at the chin. ${ }^{7,8}$ There are thus three potential advantages of using a bone substitute material with a low substitution rate alone or in combination with autogenous bone graft particles: (1) reduced volume of autogenous bone required to achieve the treatment objective; (2) reduced donor site morbidity; and (3) reduced graft resorption during remodeling. ${ }^{6,9}$

One of the best-documented bone substitute materials used for SFE is a deproteinized bovine bone mineral (DBBM) (Bio-Oss ${ }^{\circledR}$, Geistlich Pharma, Wolhusen, Switzerland), a porous bovine-derived bone mineral with osteoconductive properties. ${ }^{10} \mathrm{DBBM}$ is available in two different particle sizes: $0.25-1 \mathrm{~mm}$ and 1-2 mm. It has been speculated that utilization of the larger particles for SFE would leave more space for vascular ingrowth, which is essential for bone formation in the augmented volume. ${ }^{11}$ Therefore, large particles of DBBM have been recommended for SFE. However, there are no scientific data to support this recommendation.

A recent case series of 10 patients ${ }^{12}$ compared small and large DBBM particles for SFE using a split-mouth design and showed no difference in bone volume based on trephine biopsies harvested after 6 to 9 months of healing. However, no information is available on poten- tial differences in the course of bone healing and on the process of osseointegration of dental implants in the augmented volume.

Pull-out strength as a measure of the stability of dental implants after SFE was compared with boneto-implant contact (BIC) in experimental studies in sheep using particulated autograft or DBBM as grafting materials. ${ }^{13,14}$ However, the implant stability quotient (ISQ) has gained increased popularity in recent years as a noninvasive method to test implant stability at implant placement, over the course of the osseointegration period, and prior to prosthetic loading. ${ }^{15}$ Experimental studies correlating ISQ and BIC have shown divergent results. ${ }^{16}$ However, this has never been investigated after SFE procedures with simultaneous placement of implants. Potentially, ISQ measurements could be valuable in determining the right time point to load an implant placed in a site where SFE has been performed. In that respect, it would be of great value to know the correlation between the ISQ values and histomorphometric data of BIC, bone density, and bone-to-DBBM contact in the entire augmented volume.

The SFE model is well established in minipigs for the comparison of the biological behavior of bone grafting materials and bone growth factors in implant dentistry. ${ }^{5,6,17-24}$ Implant stability has not previously been reported using the minipig model.

The primary objective of the present study was to study bone formation after SFE using DBBM of two different particle sizes. The secondary aim was to correlate the implant stability (ISQ) of implants placed simultaneously with SFE to BIC and bone density around the implants. The null hypothesis was that the particle size of DBBM used for maxillary SFE in minipigs with simultaneous implant placement does not influence the following parameters: (1) amount of new bone formation; (2) rate of new bone formation; (3) bone-toDBBM surface contact; (4) BIC; and (5) implant stability (ISQ values).

\section{MATERIAL AND METHODS}

The present study was approved by the veterinary authorities supervising animal experiments, Office of Agriculture, State Department of Canton Bern, Switzerland (approval no. BE39/11).

The study was performed in 10 adult Göttingen minipigs (mean weight: $53.5 \mathrm{~kg} \pm 5.3 \mathrm{~kg}[ \pm \mathrm{SD}]$ ). 


\section{Surgical Procedure}

The surgeries were performed at the Surgical Research Unit, Department of Clinical Research and Clinic for Large Animals, University of Bern, Switzerland. Each animal fasted at least 12 hours before premedication with an intramuscular injection of ketamine $20 \mathrm{mg} / \mathrm{kg}$ body weight (Narketan ${ }^{\circledR} 10,100 \mathrm{mg} / \mathrm{ml}$, Vétoquinol AG, Bern, Switzerland) and xylazine $2 \mathrm{mg} / \mathrm{kg}$ body weight (Xylapan ${ }^{\circledR} 20 \mathrm{mg} / \mathrm{ml}$, Vétoquinol AG). General anesthesia was induced by an i.v. infusion of atropine $0.05 \mathrm{mg} / \mathrm{kg}$ body weight $(1 \mathrm{mg} / \mathrm{ml}$, Sintetica S.A., Mendrisio, Switzerland) and midazolam $0.5 \mathrm{mg} / \mathrm{kg}$ body weight (Dormicum $^{\circledR}$, Roche Pharma AG, Reinach, Switzerland) through an ear vein. For intraoperative protection, eye ointment (Bepanthen ${ }^{\circledR}$, Dexpanthenol $50 \mathrm{mg} / \mathrm{g}$, Bayer Vital, Leverkusen, Germany) was applied in each eye. Endotracheal intubation was performed and a controlled respiration frequency of 12 breaths/ minute was maintained with a volume of $10 \mathrm{ml} / \mathrm{kg}$ body weight. Isoflurane 1.0-1.5\% (Forene ${ }^{\circledR}$, Abbot AG, Baar, Switzerland) was added to a mixture of oxygen and $\mathrm{N}_{2} \mathrm{O}$ (ratio 1:3).

Prior to surgery, the animals were given prophylactic antibiotics 12,000 IU/kg body weight (benzylpenicillin benzathinum 150,000 IU/ml, Duplocillin ${ }^{\circledR}$ LA, Veterinaria AG, Zürich, Switzerland) intramuscularly.
For intraoperative pain control and hemostasis, local anesthesia was injected in the surgical field (4 $\mathrm{ml}$ articaine hydrochloride/epinephrine hydrochloride $40 \mathrm{mg} / \mathrm{ml} / 0.006 \mathrm{mg} / \mathrm{ml}$, Ultracaine ${ }^{\circledR}$ D-S, SanofiAventis Deutschland GmbH, Frankfurt a.M., Germany). Through bilateral infraorbital incisions, the lateral parts of the zygomatic bodies and arches were exposed. A lateral window measuring $10 \times 10 \mathrm{~mm}$ was prepared to the maxillary sinus using round burs followed by ball-shaped diamond burs, with copious saline irrigation. The schneiderian membrane was carefully elevated using blunt dissectors. If septae were present they were removed with a round bur to create one major sinus compartment. An implant bed was prepared to accommodate an implant $\varnothing: 4.1 \mathrm{~mm}$, length: $12 \mathrm{~mm}$ (Straumann Tissue Level Implant, Standard neck, SLActive ${ }^{\circledR}$, Institut Straumann AG, Basel, Switzerland) (Figure 1A). If perforation of the schneiderian membrane occurred, the membrane was reflected additionally and a minor piece of collagen membrane (BioGide ${ }^{\circledR}$, Geistlich Pharma) was used to cover the perforation. Before implant insertion, the posterior and superior parts of the sinus cavity were grafted with large (1-2 mm) or small $(0.25-1 \mathrm{~mm})$ particles of DBBM $\left(\right.$ Bio-Oss ${ }^{\circledR}$, Geistlich Pharma) according to a randomization plan (http://www.randomization.com, seed: 16423)
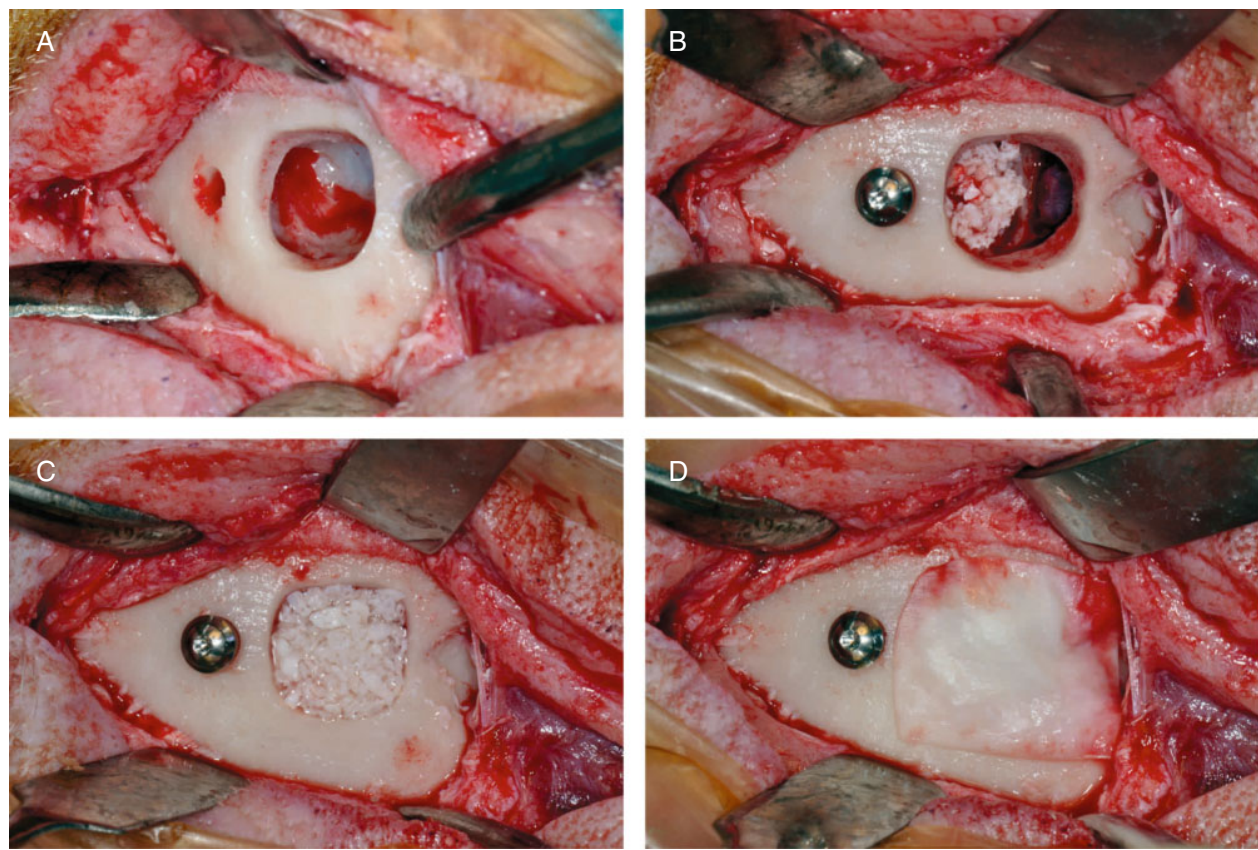

Figure 1 Surgical photos. $A$, A lateral window is prepared and the schneiderian membrane elevated. In addition, the implant bed is prepared $5 \mathrm{~mm}$ posterior to the lateral window. $B$, DBBM particles are grafted into the posterior and medial parts of the created volume in the maxillary sinus cavity before placement of a dental implant. $C$, The rest of the created volume is augmented. $D$, A collagen membrane is adapted to cover the lateral window. 
(Figure 1B). After implant insertion, implant stability (ISQ) was measured using an Osstell Mentor ${ }^{\circledR}$ (Osstell AB, Gothenburg, Sweden). Three measurements were recorded in the horizontal direction followed by three in the vertical direction. The rest of the created space was grafted (Figure 1C) and the lateral window was covered with a collagen membrane (BioGide ${ }^{\circledR}$, Geistlich Pharma) (Figure 1D). The soft tissues were closed in multiple layers using resorbable sutures (Vicryl ${ }^{\circledR}$ 3-0 and 2-0, Ethicon, Norderstedt, Germany). A fentanyl patch (Durogesic ${ }^{\circledR}$ TTS $50 \mu \mathrm{g} / \mathrm{h}$, Janssen-Cilag AG, Baar, Switzerland) was applied for postoperative pain control and antibiotic administration was repeated twice postoperatively, on day 3 and day 6 .

The animals were checked daily for the first postoperative week for signs of infection by the chief veterinarian of the Surgical Research Unit.

\section{Healing Periods}

The 10 animals were divided into 2 groups of 5 animals, which were allowed to heal for 6 weeks and 12 weeks, respectively.

At the end of each designated healing period, the animals were sacrificed by induction of deep anesthesia followed by withdrawal of the entire blood volume. The head was separated from the body and the soft tissue and mandibles were removed. ISQ measurements were repeated. The skull was divided at the midline using a saw, and two blocks, each containing one entire sinus, were harvested for histologic preparation. The pins inserted for ISQ measurements were left in place for later localization purposes.

\section{Histologic Preparation}

The two maxillary blocks were fixed in $10 \%$ neutral buffered formalin combined with $1 \% \mathrm{CaCl}_{2}$ for two weeks. Each block was dehydrated and embedded in methyl methacrylate. Using a low-speed diamond saw with copious water cooling, all defects were sectioned in the axis of the implant, yielding 10-15 consecutive undecalcified sections $(\sim 600 \mu \mathrm{m}$ in thickness). Four sections per defect (two sections containing the implant, one section anterior, and one section posterior to the implant) were mounted on opaque Plexiglas with acrylic glue and ground to a final thickness of $\sim 200 \mu \mathrm{m}$. Finally, the sections were superficially stained with toluidine blue. $^{25}$

\section{Histomorphometric Evaluation}

All histomorphometric data were collected by two experienced examiners who were blinded to the treatment modalities.

BIC was evaluated on the two most central sections of the implant as the percentage (\%) of the total implant surface covered with bone and as the percentage of the implant surface in the augmented sinus volume covered with newly formed bone by counting intersections using a square grid (distance between test lines: $0.063 \mathrm{~mm}$ ) at a magnification of $\times 160$. The thickness of the lateral sinus wall (WT) (in $\mathrm{mm}$ ) was measured directly via the microscope on each side of the implant on the same two sections. The volume fractions (\%) of newly formed bone (BV), of residual DBBM filler material (DBBMV), and of soft tissue/marrow space (ST) occupying the augmented volume were evaluated for four sections: the two sections containing the implant and the sections $5 \mathrm{~mm}$ anterior and posterior to the implant. Regions of interest (ROI) were evaluated as illustrated in Figure 2. The tissue fractions were determined by point counting directly in the microscope, using a square grid (distance between $6 \times 6$ test lines: $0.1 \mathrm{~mm}$ ) at a magnification of $\times 160$. The percentage of graft particle surface covered with newly formed bone (osteoconduction $[\mathrm{OC}]$ ) was evaluated by counting intersections using the same grid and magnification as mentioned above. $^{26}$

\section{Statistical Analysis}

The initial descriptive analysis of the data was done by drawing scatter plots for the distribution of the data sets. To detect potential differences in impact of the two different particle sizes on the parameters tested (bone volume, DBBM, BIC sinus, BIC total, OC, postoperative ISQ values) for each time point, nonparametric analysis of longitudinal data was used applying a BrunnerLanger model.

The level of significance chosen for all statistical tests was $p \leq 0.05$. Due to the small sample size and the explorative nature of this study, the $p$ values were not adjusted for multiple testing. Therefore, statistically significant findings should be interpreted as trends. The analyses of longitudinal data were performed using a software package $\mathrm{R}$ (version 2.14.1; http://www. r-project.org). 

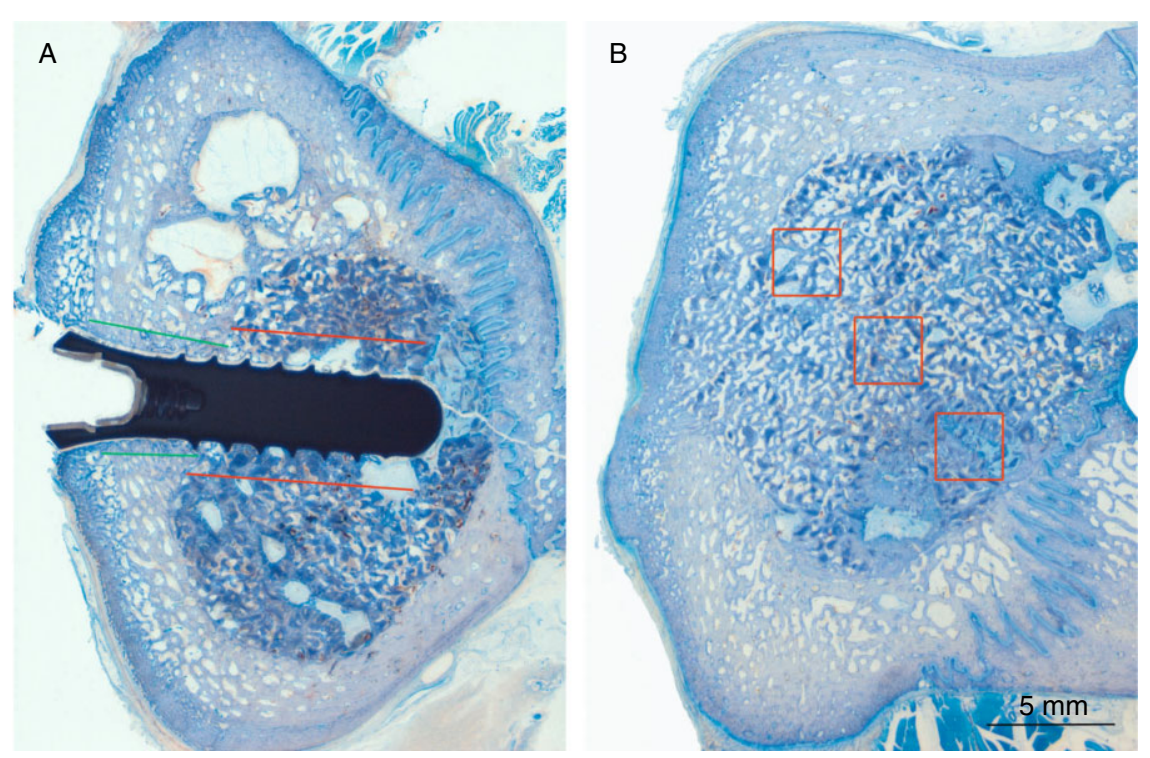

Figure 2 Histologic overview sections showing regions of interest for the histomorphometric evaluation. A, Overview, central section through the dental implant. The thickness of the original sinus wall is recorded as the average between the thickness on the mesial and on the distal aspect (green lines). In this case the sinus wall around the implant is unusually thick due to the fact that the implant was placed in the area of a former sinus septum. The red lines delineate the area where the histomorphometric analysis of bone volume, DBBM volume, and osteoconduction was performed on the two central sections including the dental implant. $B$, Overview section $5 \mathrm{~mm}$ mesial to the dental implant in the area of the former lateral window. The red squares indicate the areas where the histomorphometric evaluations were performed: a caudal-medial square, a central square, and a cranio-lateral square. In every other section the order was shifted: a cranio-medial square, a central square, and a caudal-lateral square.

\section{RESULTS}

\section{Clinical Observations}

One minipig had to be excluded at the time of surgery due to an almost complete lack of maxillary sinus, bilaterally. Therefore an additional animal was included to have $n=5$ in each test group. Perforations of the schneiderian membrane occurred in 13 of the 20 SFE procedures and were observed only in cases presenting with multiple septae. Five of 10 minipigs suffered minor nose bleeding after the surgery, all of which stopped spontaneously within the first 24 hours postoperatively. All animals healed uneventfully without any clinical signs of adverse reactions to the surgical procedure or the applied biomaterials.

\section{Histology}

Two specimens (one with large DBBM particles at 6 weeks and one with large DBBM particles at 12 weeks) had to be excluded from the analysis due to migration of the DBBM particles, presumably through the perforated sinus membrane away from the ROI (Figure 2), allowing the reformation of sinus cavities. In addition, an infection was observed on the mesial aspect of one implant (large DBBM particles, 6 weeks). In this speci- men BIC was not performed, but since the infection histologically appeared to be local and well defined, BV, DBBMV, and OC recordings were included in the final evaluation.

Often, the maxillary-zygomatic suture was surgically opened during removal of sinus septae on the medial aspect of the maxillary sinus. This allowed fibrous tissue ingrowth into the augmented volume and around the implant, displacing the DBBM particles. However, no histological signs of differences in graft displacement, in healing pattern, or in maturity of newly formed bone were observed between the two particle sizes (small vs large DBBM particles). Therefore, the histological description summarizes the qualitative tissue reactions at the two time points irrespective of DBBM particle size.

Six Weeks. Bone formation was exclusively observed extending from the sinus walls. Maturity of the newly formed bone, correspondingly, increased from the periphery of the maxillary sinus. Woven bone or no bone was observed at this time point in the center of the augmented volume including the apex of the implants, whereas the woven bone lattice in the periphery was reinforced with parallel-fibered bone. Struts of woven 

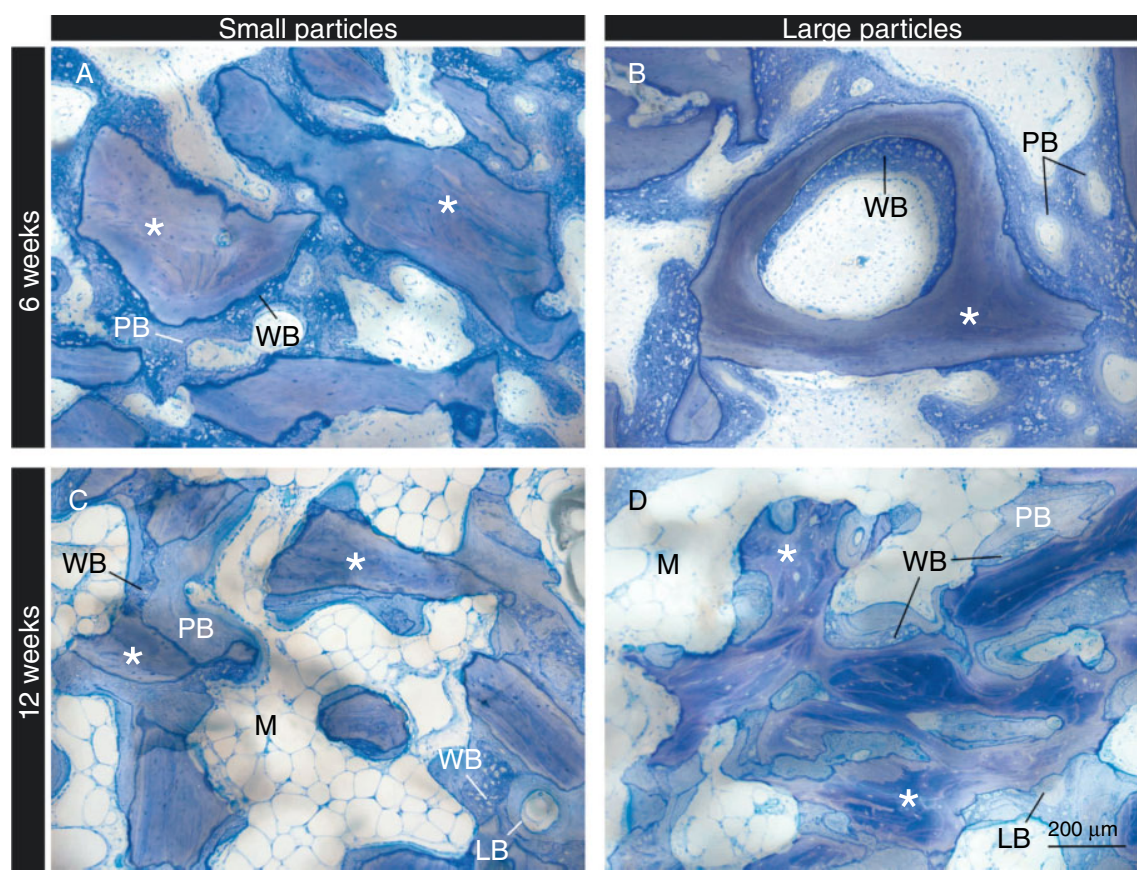

Figure 3 Bone healing around small and large DBBM $\left(^{*}\right)$ particles after 6 and 12 weeks of healing. 6 weeks: Small (A) and large (B) DBBM $\left(^{*}\right)$ particles are almost completely embedded in woven bone (WB) after 6 weeks. Limited amounts of parallel-fibered bone $(\mathrm{PB})$ are observed. The soft tissue is relatively rich in cells and free of inflammation. 12 weeks: Small (C) and large (D) DBBM $\left.{ }^{*}\right)$ particles integrated in mature parallel-fibered bone $(\mathrm{PB})$ and lamellar bone (LB) with a mature bone marrow $(\mathrm{M})$ in between. A central lattice of woven bone can still be recognized (WB).

bone reached from DBBM particle to DBBM particle, often amalgamating the complete periphery of the particles (Figure 3). Similarly, osseointegration of the implants mainly took place from the sinus wall, with woven bone extending along the implant surface (Figure 4). DBBM particles were never observed in direct contact with the implant surface. The soft tissue in between the particles and the newly formed bone had the character of a developing bone marrow, relatively poor in cells and free of inflammation. In areas where DBBM was engulfed by fibrous tissue originating from the maxillary-zygomatic suture, the particles were often completely surrounded by osteoclastic multinucleated cells showing signs of pronounced resorptive activity (Figure 5).

Twelve Weeks. Increased maturity and density of the newly formed bone was observed around the DBBM particles as well as around the implants (Figures 3 and 4). Parallel-fibered bone dominated throughout the augmented volume, and primary osteons were often seen (Figure 3). A mature bone marrow had developed (Figure 3). Multinucleated cells were often observed on the surface, wherever DBBM was exposed to the bone marrow. In contrast to the DBBM particles captured in fibrous tissue early in the healing phase (Figure 5A), these multinucleated cells did not show signs of active resorption and were hence localized on unaffected DBBM surfaces or in very shallow concavities (Figure 5B).

\section{Histomorphometry}

The main findings of the histomorphometric analysis are presented in Table 1. The total BIC and BIC within the augmented volume both increased from week 6 to week 12 in both test groups $(p<0.05)$. BV also increased from week 6 to week 12 but without reaching statistical significance $(p>0.05)$. There was a limited but significant decrease in DBBMV from week 6 to week 12 $(p<0.001)$ but no difference between the two particle sizes $(p>0.05)$. There was a statistically significant difference in OC of small and large particles of DBBM over the two time periods analyzed $(p<0.001)$.

\section{Implant Stability}

The overall ISQ values increased significantly from 70.6 at the time of implant placement to 83.3 and 84.0 after 6 and 12 weeks, respectively $(p<0.001)$ (Figure 6). There was no statistically significant correlation between initial 

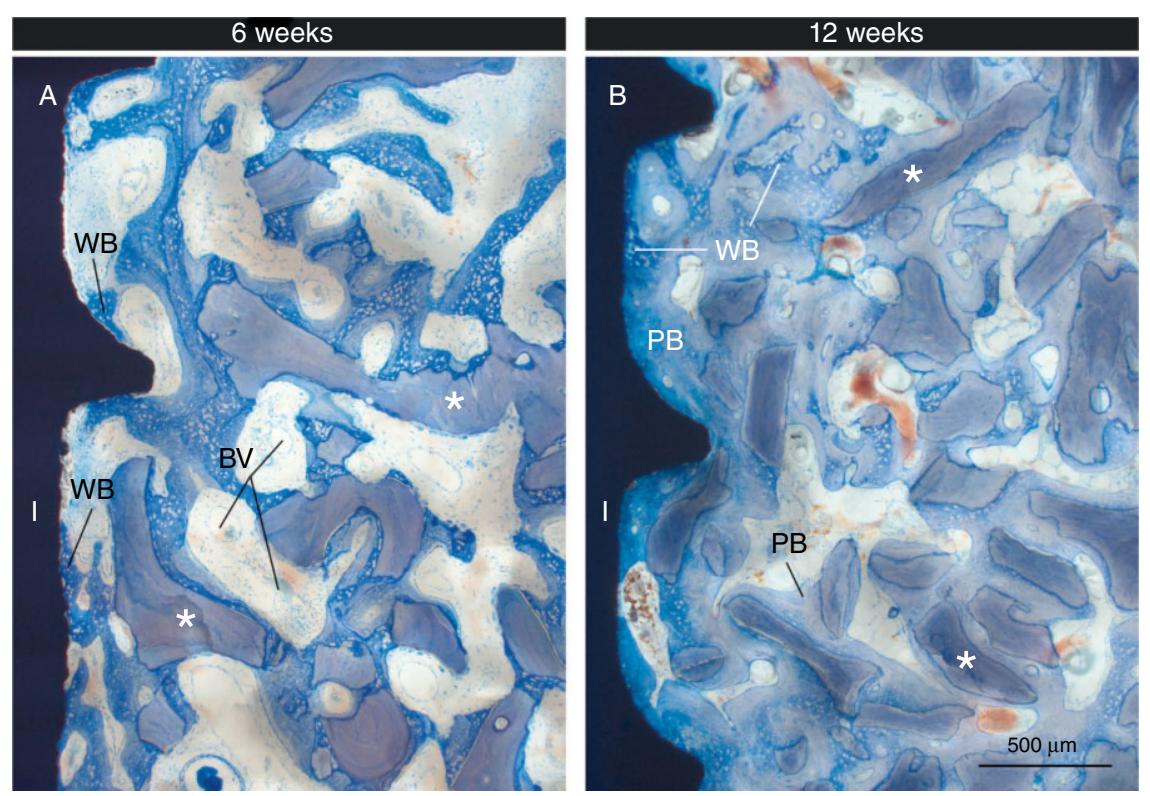

Figure 4 Osseointegration of implants. A, An implant with a chemically modified sandblasted acid-etched hydrophilic implant (I) surface partly covered with a sheet of woven bone (WB) in a maxillary sinus augmented with DBBM $\left({ }^{*}\right)$ of small size after 6 weeks of healing. Note the connection of the DBBM $\left(^{*}\right)$ particles with branches of woven bone and the high number of blood vessels (BV). $B$, After 12 weeks of healing, bone in the peri-implant area appears considerably denser and more mature, with parallel-fibered bone $\mathrm{PB})$ and lamellar bone dominating over woven bone (WB).

thickness of the sinus wall in which the implant was placed and the ISQ values at the time of implant placement and at the end of each designated healing period. Moreover, there was no statistically significant correlation between ISQ and BIC at the end of the healing periods.

\section{DISCUSSION}

The present study evaluated the influence of the particle size of DBBM on bone formation and implant stability when used for SFE in a well-documented minipig model. Within the limitations of the study design, in the initial healing phase small particle size DBBM showed marginally higher osteoconductive capacity than large particle size DBBM. However, no differences were observed in amount and speed of bone formation, BIC, or implant stability between the two test groups. The null hypothesis could therefore be accepted.

Surgically and histologically the animal model showed challenges that have not been reported
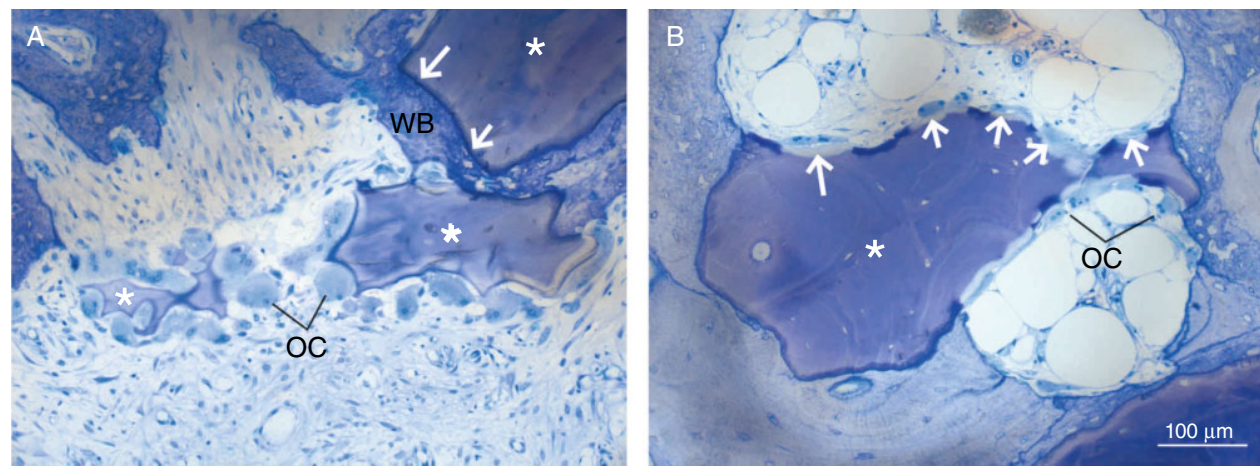

Figure 5 Degradation of DBBM. A, 6 weeks. Three DBBM $\left(^{*}\right)$ particles in an area with early fibrous ingrowth. One is covered with newly formed woven bone (WB), with what appears to be an intact particle surface (arrows). The two others, however, are captured in a cell-rich soft tissue, showing signs of pronounced resorption through multiple multinucleated osteoclast-like cells (OC). $B, 12$ weeks. DBBM $\left(^{*}\right)$ particles are well integrated in bone and mature bone marrow. Where the DBBM surface is exposed to the bone marrow, multiple multinucleated osteoclast-like cells (OC) are observed. However, they are situated on a flat surface or in shallow concavities (arrows), unlike in Figure 5A. 


\section{TABLE 1 Results of Histomorphometric Analysis and Implant Stability Measurements}

\begin{tabular}{|c|c|c|c|c|c|c|c|c|c|}
\hline Healing Time & Particle Size & WT $\mathrm{mm}$ & ISQ po & ISQ sacr & $\mathrm{BIC}$ sinus $\%$ & BIC tot $\%$ & BV \% & DBBMV \% & OC $\%$ \\
\hline \multirow[t]{8}{*}{6 weeks } & Small & & & & & & & & \\
\hline & Mean & 2.4 & 67.7 & 83.5 & 15.4 & 29.2 & 39.0 & 25.1 & 80.1 \\
\hline & Median & 2.6 & 66.0 & 84.0 & 15.4 & 28.3 & 39.4 & 26.2 & 79.6 \\
\hline & SD & 0.6 & 8.1 & 1.7 & 0.2 & 3.5 & 6.3 & 2.6 & 2.9 \\
\hline & Large & & & & & & & & \\
\hline & Mean & 2.7 & 68.2 & 81.8 & 18.7 & 35.1 & 40.0 & 24.6 & 71.0 \\
\hline & Median & 2.8 & 68.5 & 83.0 & 21.2 & 35.3 & 36.5 & 25.1 & 72.2 \\
\hline & $\mathrm{SD}$ & 0.5 & 7.0 & 3.6 & 4.8 & 3.6 & 7.6 & 1.9 & 5.2 \\
\hline \multirow[t]{8}{*}{12 weeks } & Small & & & & & & & & \\
\hline & Mean & 3.2 & 73.7 & 83.4 & 35.3 & 53.5 & 44.3 & 21.3 & 83.1 \\
\hline & Median & 3.0 & 75.5 & 85.5 & 39.9 & 53.8 & 45.7 & 21.8 & 80.6 \\
\hline & SD & 0.7 & 4.9 & 4.3 & 16.3 & 17.9 & 3.2 & 3.6 & 5.0 \\
\hline & Large & & & & & & & & \\
\hline & Mean & 3.2 & 72.1 & 84.5 & 32.2 & 51.5 & 45.1 & 19.8 & 82.3 \\
\hline & Median & 2.9 & 74.0 & 85.5 & 32.8 & 49.7 & 46.3 & 20.9 & 82.1 \\
\hline & $\mathrm{SD}$ & 1.1 & 6.6 & 2.5 & 8.4 & 14.5 & 4.9 & 3.5 & 2.7 \\
\hline
\end{tabular}

WT, sinus wall thickness; ISQ, implant stability quotient; po, immediately after implant placement and sinus grafting; sacr, at sacrifice; BIC, bone-toimplant contact; $\mathrm{BV}$, bone volume: fraction of newly formed bone in the augmented volume; DBBMV, DBBM volume: fraction of remaining DBBM in the augmented volume; OC, osteoconduction: fraction of DBBM surface covered with newly formed bone.

previously. The frequent finding of septae dividing the maxillary sinus into multiple compartments led to a high incidence of sinus membrane perforations and opening of the maxillary-zygomatic suture causing ingrowth of fibrous tissue around the implants. This anatomical variation might be related to the different origin of animals previously used. ${ }^{6,17,19-24}$ However, BIC values after 6 and 12 weeks were comparable or even higher than in previous studies using the same model. ${ }^{17,19,22}$ Due to the very variable but most often limited size of the
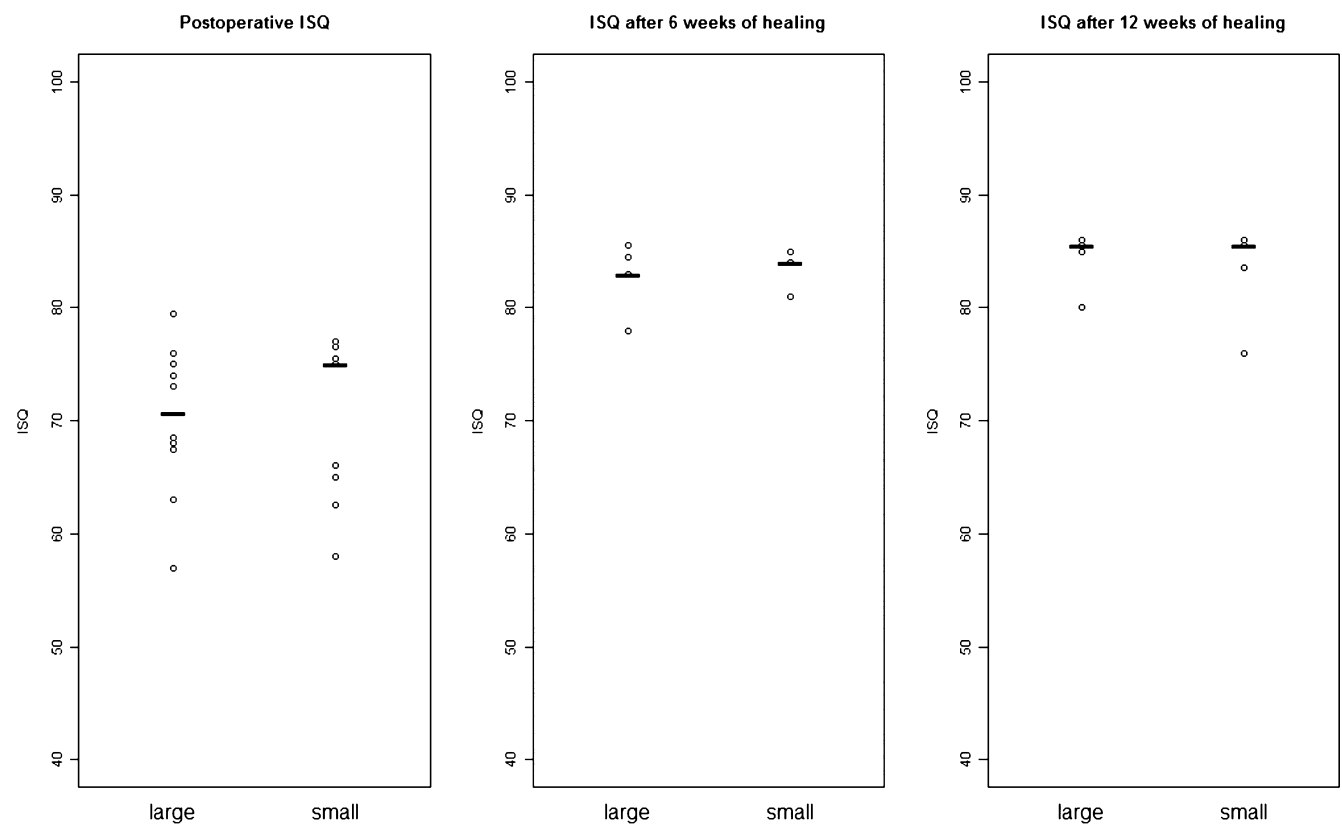

Figure 6 Implant stability. Point plots of implant stability quotient (ISQ) values immediately after implant placement, after 6 weeks of healing, and after 12 weeks of healing. Horizontal lines represent median values. 
sinus cavity, the same amount of DBBM (by weight) could not be applied in all surgical sites. The residual amount of non-grafted DBBM was weighed. However, since the complete augmented volume was not analyzed, these data are not reported here.

The volume of newly formed bone increased between 6 and 12 weeks in accordance with previous studies in the maxillary sinus of minipigs. ${ }^{18,24}$ Bone formation was not influenced by the particle size of DBBM, which is in agreement with the only human study so far comparing small and large particle size DBBM for SFE. ${ }^{12}$ Moreover, the bone volume after 6 and 12 weeks and the relation between the volume fractions of bone, DBBM and soft tissue lie within the same range as previous studies in the mandibles of minipigs, ${ }^{27,28}$ with $35-50 \%$ bone, 25-35\% DBBM, and 30-40\% soft tissue, suggesting a steady state to establish between the different tissue compartments during the course of healing.

Pallesen and colleagues reported an inverse relationship between particle size and volume of newly formed bone when small and large size particulated autografts were used in standardized calvarial defects in rabbits. ${ }^{29}$ In contrast to DBBM, autografts contain bone growth factors. ${ }^{30}$ When particle size decreases, the surface area increases, whereby the presentation of growth factors may be expected to increase correspondingly. Two rabbit studies on guided bone regeneration ${ }^{31}$ and $\mathrm{SFE}^{32}$ reported more new bone formation with small DBBM particles than with large ones. In contrast to the present study, the volume of graft particles in the two experimental studies was significantly higher in the large particle groups, which probably allowed less volume to be occupied by newly formed bone.

A limited but significant reduction in volume of DBBM irrespective of particle size was observed from week 6 to week 12. The same tendency has been reported after SFE in humans, ${ }^{9}$ experimentally in an identical animal model, ${ }^{24}$ and in the mandibles of minipigs. ${ }^{27,28}$ Only one study in minipigs has reported pronounced resorption of DBBM after 6 months of healing. ${ }^{17}$ In general, DBBM is considered to be clinically nonresorbable, without signs of significant degradation up to 11 years after SFE. ${ }^{33}$ However, an overall reduction of the entire augmented volume is well known after SFE procedures. It has mainly been studied using twodimensional panoramic radiographs and takes place irrespective of the grafting protocol until it stabilizes approximately 1 year postoperatively. ${ }^{34-36}$
The degree of volume reduction is dependent on the resorbability of the grafting materials used. In humans, volume reductions of $28 \%$ to $38 \%$ have been documented using autografts or $\beta$-tricalcium phosphate, respectively. ${ }^{34}$ When DBBM was used, on the other hand, the volume reduction was below $10 \% \cdot{ }^{35,36}$ In minipigs, it was demonstrated three-dimensionally using CT scans that the reduction of graft volume after SFE was directly related to the ratio between particulated autografts and DBBM. ${ }^{6}$ Degradation of DBBM was not influenced by the size of the particles applied in the present study. For autografts, on the other hand, resorption has been shown to increase as the size of the particles decreases. ${ }^{29}$ It is plausible that an almost nonresorbable material stays nonresorbable irrespective of the particle size, whereas resorption of degradable materials accelerates as the exposed area of the materials increases.

An interesting finding was the pronounced resorption of DBBM in a few selected areas where fibrous tissue from the maxillary-zygomatic suture was allowed to grow into the augmented volume (Figure 5A). A similar finding was reported in a recent study using a porcine calvarial model. ${ }^{37}$ From the present study it seems likely that DBBM particles hosted in an osseous environment may undergo only limited resorption whenever exposed to the marrow cavity, whereas particles captured in fibrous tissue early in the healing phase are at significant risk of complete degradation. It may be speculated that differences in protein adsorbed to the DBBM alter the chemotactic effect of the biomaterial surface and thus the type and function of the cells that colonize the surface. ${ }^{38}$

DBBM has been shown to be highly osteoconductive in previous human ${ }^{39,40}$ and animal experimental studies. ${ }^{24,27}$ The effect of particle size on osteoconductive properties of DBBM has not been investigated previously. In the early healing phase, small DBBM particles demonstrated higher osteoconductive capacity than large particles. In addition, as particle size decreased, the absolute surface area increased, leaving more DBBM to be covered with bone. Therefore, based on the present findings, a larger area of DBBM should be expected to be "osseointegrated" when using small-sized DBBM particles.

BIC has previously been studied after SFE using DBBM alone with simultaneous implant placement in humans $^{39}$ and in animals. ${ }^{5,13,18-20}$ The highest BIC value 
was reported in the human study (66\%). ${ }^{39}$ However, this value was achieved after 8 months of healing and is therefore difficult to compare directly with the present study. Five animal studies contain BIC data after 6 and/or 12 weeks of healing, as in the present study. After 6 weeks, Liu and colleagues presented 13\% BIC in the apical part of the implants placed in minipigs. ${ }^{20}$ However, the "apical part of the implant" was not defined, which makes comparisons to the present study difficult. Fürst and colleagues reported $3 \%$ and $7 \%$ BIC after 6 and 12 weeks, respectively, in minipigs. ${ }^{18}$ That study used implants with a machined surface, which may explain the low BIC values in the early phases of healing. In minipigs, sheep, and dogs, moderately rough implant surfaces demonstrated BIC values of $14 \%, 27 \%$, and $34 \%$, respectively, after 12 weeks of healing., ${ }^{5,13,19}$ The present study documented BIC values of $15 \%$ and $19 \%$ after 6 weeks and of 35\% and 32\% after 12 weeks, respectively. This compares well to the previous studies, especially in the early healing period. However, the present study was the first to use a chemically modified hydrophilic sandblasted and acid-etched implant surface, which has been shown to accelerate bone formation around test implants during initial wound healing. ${ }^{41}$ Another explanation for the high amount of new bone formation and the high BIC may be the relatively narrow sinus anatomy observed in the present study. The role of the anatomy has not been addressed in previous minipig studies, but from the figures it seems that the maxillary sinus cavities might have been larger than in the present study. Bone healing is known to take place from the existing bone walls, ${ }^{24}$ and with reduced distance between the sinus walls, the osteogenic capacity of the sinus may increase. ${ }^{42}$

No correlation was found between initial sinus wall thickness and primary implant stability (ISQ). This is in accordance with a clinical study of implant stability after transcrestal SFE with simultaneous implant placement in humans. ${ }^{43}$ Primary implant stability is dependent not only on the thickness of the bone into which the implant is placed but also on the bone density, the thread configuration and shape of the implant, and the presence of an implant neck. Therefore, all these factors should be taken into consideration when making the clinical decision to perform a one-stage or a two-stage procedure.

To the authors' knowledge, this is the first study to compare ISQ with BIC values after SFE procedures. No correlation could be observed between BIC at 6 or 12 weeks and secondary implant stability (ISQ). This is in accordance with previous animal studies of implants placed in nonaugmented bone. ${ }^{44-46}$ During healing, primary stability is reduced due to remodeling of the implant bed, while increasing osseointegration (BIC) ensures the secondary stability of the implant. ${ }^{47}$ Therefore, two almost identical ISQ values at implant insertion and at loading represent two very different biologic scenarios at the implant surface. ${ }^{48}$ It is therefore advisable to focus not only on the absolute ISQ value but also on the longitudinal development of the ISQ value over the course of healing. Decreasing ISQ values after the very early healing phase (3-4 weeks), on the other hand, might require an extended healing period until loading, or might even be predictive of implant failure. ${ }^{16}$

\section{CONCLUSION}

Within the limitations of the present animal experimental study it is concluded that small and large particle size DBBM perform equally predictably when used for SFE with simultaneous implant placement.

\section{ACKNOWLEDGMENTS}

The authors wish to thank Mrs. S. Owusu, Mrs. M. Rüfenacht, and Mr. D. Reist, Robert K. Schenk Laboratory for Oral Histology, School of Dental Medicine, University of Bern, Bern, Switzerland, for their valuable assistance in preparing the histologic sections and performing the histomorphometric analysis; Ms. C. Moser, Department of Oral Surgery and Stomatology, School of Dental Medicine, University of Bern, Bern, Switzerland, for her professional help during all surgeries, and the entire staff at the Surgical Research Unit ESI, Department of Clinical Research and Clinic for Large Animals, University of Bern, Switzerland, for excellent handling of the animals. The authors also thank Lukas Martig, Institute of Mathematical Statistics and Actuarial Science, University of Bern, Bern, Switzerland, for assistance with the statistical analyses.

The study was funded by the Department of Oral Surgery and Stomatology, University of Bern, Switzerland, and by Geistlich Pharma, Wolhusen, Switzerland.

The DBBM (Bio-Oss ${ }^{\circledR}$ ) and collagen membranes (BioGide ${ }^{\circledR}$ ) were provided free of charge by Geistlich Pharma, Wolhusen, Switzerland, and the dental implants (Straumann Tissue Level Implants, Standard neck, SLActive ${ }^{\circledR}$ ) were provided free of charge by Institut Straumann AG, Basel, Switzerland. 
The authors report no financial relationships related to any products involved in this study.

\section{REFERENCES}

1. Esposito M, Cannizzaro G, Soardi E, et al. Posterior atrophic jaws rehabilitated with prostheses supported by $6 \mathrm{~mm}$-long, $4 \mathrm{~mm}$-wide implants or by longer implants in augmented bone. Preliminary results from a pilot randomised controlled trial. Eur J Oral Implantol 2012; 5:19-33.

2. Hagi D, Deporter DA, Pilliar RM, Arenovich T. A targeted review of study outcomes with short $(<$ or $=7 \mathrm{~mm})$ endosseous dental implants placed in partially edentulous patients. J Periodontol 2004; 75:798-804.

3. Jensen SS, Terheyden H. Bone augmentation procedures in localized defects in the alveolar ridge: clinical results with different bone grafts and bone-substitute materials. Int J Oral Maxillofac Implants 2009; 24 (Suppl):218-236.

4. Johansson B, Grepe A, Wannfors K, Hirsch JM. A clinical study of changes in the volume of bone grafts in the atrophic maxilla. Dentomaxillofac Radiol 2001; 30:157-161.

5. Schlegel KA, Fichtner G, Schultze-Mosgau S, Wiltfang J. Histologic findings in sinus augmentation with autogenous bone chips versus a bovine bone substitute. Int J Oral Maxillofac Implants 2003; 18:53-58.

6. Jensen T, Schou S, Svendsen PA, et al. Volumetric changes of the graft after maxillary sinus floor augmentation with BioOss and autogenous bone in different ratios: a radiographic study in minipigs. Clin Oral Implants Res 2012; 23:902-910.

7. Cricchio G, Lundgren S. Donor site morbidity in two different approaches to anterior iliac crest bone harvesting. Clin Implant Dent Relat Res 2003; 5:161-169.

8. Clavero J, Lundgren S. Ramus or chin grafts for maxillary sinus inlay and local onlay augmentation: comparison of donor site morbidity and complications. Clin Implant Dent Relat Res 2003; 5:154-160.

9. Hallman M, Lundgren S, Sennerby L. Histologic analysis of clinical biopsies taken 6 months and 3 years after maxillary sinus floor augmentation with $80 \%$ bovine hydroxyapatite and $20 \%$ autogenous bone mixed with fibrin glue. Clin Implant Dent Relat Res 2001; 3:87-96.

10. Jensen SS, Aaboe M, Pinholt EM, Hjorting-Hansen E, Melsen F, Ruyter IE. Tissue reaction and material characteristics of four bone substitutes. Int J Oral Maxillofac Implants 1996; 11:55-66.

11. Carano RA, Filvaroff EH. Angiogenesis and bone repair. Drug Discov Today 2003; 8:980-989.

12. Chackartchi T, Iezzi G, Goldstein $\mathrm{M}$, et al. Sinus floor augmentation using large $(1-2 \mathrm{~mm})$ or small $(0.25-1 \mathrm{~mm})$ bovine bone mineral particles: a prospective, intraindividual controlled clinical, micro-computerized tomography and histomorphometric study. Clin Oral Implants Res 2011; 22:473-480.
13. Haas R, Donath K, Fodinger M, Watzek G. Bovine hydroxyapatite for maxillary sinus grafting: comparative histomorphometric findings in sheep. Clin Oral Implants Res 1998; 9:107-116.

14. Haas R, Mailath G, Dortbudak O, Watzek G. Bovine hydroxyapatite for maxillary sinus augmentation: analysis of interfacial bond strength of dental implants using pull-out tests. Clin Oral Implants Res 1998; 9:117-122.

15. Bornstein MM, Hart CN, Halbritter SA, Morton D, Buser D. Early loading of nonsubmerged titanium implants with a chemically modified sand-blasted and acid-etched surface: 6-month results of a prospective case series study in the posterior mandible focusing on peri-implant crestal bone changes and implant stability quotient (ISQ) values. Clin Implant Dent Relat Res 2009; 11:338-347.

16. Sennerby L, Meredith N. Implant stability measurements using resonance frequency analysis: biological and biomechanical aspects and clinical implications. Periodontol 2000 2008; 47:51-66.

17. Terheyden H, Jepsen S, Moller B, Tucker MM, Rueger DC. Sinus floor augmentation with simultaneous placement of dental implants using a combination of deproteinized bone xenografts and recombinant human osteogenic protein-1. A histometric study in miniature pigs. Clin Oral Implants Res 1999; 10:510-521.

18. Fürst G, Gruber R, Tangl S, et al. Sinus grafting with autogenous platelet-rich plasma and bovine hydroxyapatite. A histomorphometric study in minipigs. Clin Oral Implants Res 2003; 14:500-508.

19. Jensen T, Schou S, Gundersen HJ, Forman JL, Terheyden H, Holmstrup P. Bone-to-implant contact after maxillary sinus floor augmentation with Bio-Oss and autogenous bone in different ratios in mini pigs. Clin Oral Implants Res 2013; 24: 635-644. DOI: 10.1111/j.1600-0501.2012.02438.x.

20. Liu Y, Springer IN, Zimmermann CE, et al. Missing osteogenic effect of expanded autogenous osteoblast-like cells in a minipig model of sinus augmentation with simultaneous dental implant installation. Clin Oral Implants Res 2008; 19:497-504.

21. Roldan JC, Jepsen S, Schmidt C, et al. Sinus floor augmentation with simultaneous placement of dental implants in the presence of platelet-rich plasma or recombinant human bone morphogenetic protein-7. Clin Oral Implants Res 2004; 15:716-723.

22. Roldan JC, Knueppel H, Schmidt C, Jepsen S, Zimmermann C, Terheyden H. Single-stage sinus augmentation with cancellous iliac bone and anorganic bovine bone in the presence of platelet-rich plasma in the miniature pig. Clin Oral Implants Res 2008; 19:373-378.

23. Gruber RM, Ludwig A, Merten HA, Pippig S, Kramer FJ, Schliephake H. Sinus floor augmentation with recombinant human growth and differentiation factor-5 (rhGDF-5): a pilot study in the Goettingen miniature pig comparing 
autogenous bone and rhGDF-5. Clin Oral Implants Res 2009; 20:175-182.

24. Busenlechner D, Huber CD, Vasak C, Dobsak A, Gruber R, Watzek G. Sinus augmentation analysis revised: the gradient of graft consolidation. Clin Oral Implants Res 2009; 20: 1078-1083.

25. Schenk RK, Olah AJ, Herrmann W. Preparation of calcified tissues for light microscopy. Amsterdam: Elsevier, 1984.

26. Weibel ER. Stereological Methods, Vol. I: practical methods for biological morphometry. New York: Academic Press, 1979.

27. Jensen SS, Bornstein MM, Dard M, Bosshardt DD, Buser D. Comparative study of biphasic calcium phosphates with different HA/TCP ratios in mandibular bone defects. A longterm histomorphometric study in minipigs. J Biomed Mater Res B Appl Biomater 2009; 90:171-181.

28. Jensen SS, Broggini N, Hjorting-Hansen E, Schenk R, Buser D. Bone healing and graft resorption of autograft, anorganic bovine bone and beta-tricalcium phosphate. A histologic and histomorphometric study in the mandibles of minipigs. Clin Oral Implants Res 2006; 17:237-243.

29. Pallesen L, Schou S, Aaboe M, Hjorting-Hansen E, Nattestad A, Melsen F. Influence of particle size of autogenous bone grafts on the early stages of bone regeneration: a histologic and stereologic study in rabbit calvarium. Int J Oral Maxillofac Implants 2002; 17:498-506.

30. Schliephake H. Bone growth factors in maxillofacial skeletal reconstruction. Int J Oral Maxillofac Surg 2002; 31: 469-489.

31. Zhou X, Zhang Z, Li S, Bai Y, Xu H. Osteoconduction of different sizes of anorganic bone particles in a model of guided bone regeneration. Br J Oral Maxillofac Surg 2011; 49:37-41.

32. Xu H, Shimizu Y, Asai S, Ooya K. Experimental sinus grafting with the use of deproteinized bone particles of different sizes. Clin Oral Implants Res 2003; 14:548-555.

33. Mordenfeld A, Hallman M, Johansson CB, Albrektsson T. Histological and histomorphometrical analyses of biopsies harvested 11 years after maxillary sinus floor augmentation with deproteinized bovine and autogenous bone. Clin Oral Implants Res 2010; 21:961-970.

34. Zijderveld SA, Schulten EA, Aartman IH, ten Bruggenkate CM. Long-term changes in graft height after maxillary sinus floor elevation with different grafting materials: radiographic evaluation with a minimum follow-up of 4.5 years. Clin Oral Implants Res 2009; 20:691-700.

35. Hallman M, Hedin M, Sennerby L, Lundgren S. A prospective 1-year clinical and radiographic study of implants placed after maxillary sinus floor augmentation with bovine hydroxyapatite and autogenous bone. J Oral Maxillofac Surg 2002; 60:277-284.
36. Mordenfeld A, Albrektsson T, Hallman M. A 10-year clinical and radiographic study of implants placed after maxillary sinus floor augmentation with an 80:20 mixture of deprotenized bovine bone and autogenous bone. Clin Implant Dent Relat Res. DOI: 10.1111/cid.12008.

37. Busenlechner D, Tangl S, Arnhart C, et al. Resorption of deproteinized bovine bone mineral in a porcine calvaria augmentation model. Clin Oral Implants Res 2012; 23:95-99.

38. Balloni S, Calvi EM, Damiani F, et al. Effects of titanium surface roughness on mesenchymal stem cell commitment and differentiation signaling. Int J Oral Maxillofac Implants 2009; 24:627-635.

39. Lindgren C, Sennerby L, Mordenfeld A, Hallman M. Clinical histology of microimplants placed in two different biomaterials. Int J Oral Maxillofac Implants 2009; 24:1093-1100.

40. Cordaro L, Bosshardt DD, Palattella P, Rao W, Serino G, Chiapasco M. Maxillary sinus grafting with Bio-Oss or Straumann Bone Ceramic: histomorphometric results from a randomized controlled multicenter clinical trial. Clin Oral Implants Res 2008; 19:796-803.

41. Buser D, Broggini N, Wieland M, et al. Enhanced bone apposition to a chemically modified SLA titanium surface. J Dent Res 2004; 83:529-533.

42. Soardi CM, Spinato S, Zaffe D, Wang HL. Atrophic maxillary floor augmentation by mineralized human bone allograft in sinuses of different size: an histologic and histomorphometric analysis. Clin Oral Implants Res 2011; 22:560-566.

43. Lai HC, Zhang ZY, Wang F, Zhuang LF, Liu X. Resonance frequency analysis of stability on ITI implants with osteotome sinus floor elevation technique without grafting: a 5-month prospective study. Clin Oral Implants Res 2008; 19:469-475.

44. Al-Nawas B, Groetz KA, Goetz H, Duschner H, Wagner W. Comparative histomorphometry and resonance frequency analysis of implants with moderately rough surfaces in a loaded animal model. Clin Oral Implants Res 2008; 19:1-8.

45. Schliephake H, Sewing A, Aref A. Resonance frequency measurements of implant stability in the dog mandible: experimental comparison with histomorphometric data. Int J Oral Maxillofac Surg 2006; 35:941-946.

46. Abrahamsson I, Linder E, Lang NP. Implant stability in relation to osseointegration: an experimental study in the Labrador dog. Clin Oral Implants Res 2009; 20:313-318.

47. Han J, Lulic M, Lang NP. Factors influencing resonance frequency analysis assessed by Osstell mentor during implant tissue integration: II. Implant surface modifications and implant diameter. Clin Oral Implants Res 2010; 21:605-611.

48. Rasmusson L, Thor A, Sennerby L. Stability evaluation of implants integrated in grafted and nongrafted maxillary bone: a clinical study from implant placement to abutment connection. Clin Implant Dent Relat Res 2012; 14:61-66. 